\title{
Topic 17: High-Performance Bioinformatics
}

\author{
Craig A. Stewart, Michael Schroeder, \\ Concettina Guerra, and Konagaya Akihiko \\ Topic Chairs
}

High performance computational biology and bioinformatics are increasingly required to extract valuable biological and biomedical knowledge from everincreasing biological data. New computational techniques and new theoretical models are required to simulate complex biological behavior of biological systems. Topic 17 focuses on high-performance and high-throughput computing necessary for management of biological data, extraction of meaning from biological data and using such data in modeling and simulation of biological systems.

Five papers were accepted for Topic 17 this year:

Multidimensional Dynamic Programming for Homology Search on Distributed Systems by Shingo Masuno, Tsutomu Maruyama, Yoshiki Yamaguchi, and Akihiko Konagaya describes a computation method for multidimensional dynamic programming on distributed systems. This paper makes use of FPGA systems in novel and interesting ways, demonstrating the utility that many people expect to see in FPGA-based systems in biocomputing in the future.

Load balancing and Parallel Multiple Sequence Alignment with Tree Accumulation by Guangming Tan proposes a load balancing strategy for parallelizing tree accumulation in progressive alignment in the widely used package ClustalW, reducing overall running time and achieving reasonable speedups.

ZIB Structure Prediction Pipeline: Composing a Complex Biological Workflow through Web Services by Patric May, Hans-Christian Ehrlich, and Thomas Steinke presents status of their efforts for the realization of an automated protein prediction pipeline as an example for a complex biological workflow scenario in a Grid environment based on Web services. As grid computing evolves, many leading experts believe the use of standards-based web services to be of particular value in bioinformatics.

Evaluation of Parallel Paradigms on Anisotropic Nonlinear Diffusion by S. Tabik, E.M. Garzn, I. Garca, and J. J. Fernndez discusses the parallel implementation of Anisotropic Nonlinear Diffusion, a powerful noise reduction technique in the field of computer vision. This technique is applied to the problem of analysis of $3 \mathrm{D}$ images, an important problem in high performance computational biology and bioinformatics.

Improving the Research Environment of High Performance Computing for Non-Cluster Experts Based on Knoppix Instant Computing Technology by Fumikazu Konishi, Manabu Ishii, Shingo Ohki, Yusuke Hamano, Shuichi Fukuda, and Akihiko Konagaya presents an approach for instant computing using Knoppix technology that can allow even a non-computer specialist to easily construct and operate a Beowulf cluster. The application InterProScan (from the Euro- 
pean Bioinformatics Institute) is used as a demonstration of the value of Knoppix Instant Computing Technology in bioinformatics.

These papers represent the very high-quality submissions received for the topic High-Performance Bioinformatics. The contribution of Shingo Masuno, Tsutomu Maruyama, Yoshiki Yamaguchi, and Akihiko Konagaya is in particular noted as a distinguished contribution. The organizers of the High-Performance Bioinformatics topic would like to thank all authors who submitted papers, the paper review committee, and the Euro-Par 2006 conference organizers. 